\title{
SPECIALIZING RDFS : SEE ALSO IN SEMANTIC WEB
}

\author{
Farzam Matinfar $^{1}$ and Mohammadali Nematbakhsh ${ }^{2}$ \\ Department of Computer Engineering, University of Isfahan, Isfahan \\ ${ }^{1}$ f.matinfardeng.ui.ac.ir, ${ }^{2}$ nematbakhsh@eng.ui.ac.ir
}

\begin{abstract}
One of the principles of Web of Data is linking resources to related ones. The rdfs:seeAlso link plays an important role in interlinking datasets and web resources.W3C defines rdfs:seeAlso to provide additional information about subject resources by interlinking datasets. This definition is general and can have various interpretations and implementations. In this paper, we tackle the problem of specializing the rdfs:seeAlso link by identifying more specific definitions and patterns that can assist individuals and organization in recognizing the target web resources that provide additional information about the subject resource. We investigate this problem in information retrieval applications and specify the desired patterns by probing databases that traditionally use seeAlso in their structure and referencing system. The structure of described databases and systems are similar to the structure of Web of Data. The provided usages of seeAlso show that many of the explained patterns are common that can be applied in Web of Data. We have conducted an empirical study to analyse the comprehensiveness of several patterns in traditional web. The results show that the investigated patterns constitute a significant portion of seeAlso usages.
\end{abstract}

\section{KEYWORDS}

Information Retrieval, Library Science, Semantic Web, Linked Data

\section{INTRODUCTION}

The traditional World Wide Web is a collection of unstructured documents that are interlinked with hyperlinks which do not provide the explicit meaning between documents. Hence, the relations between documents can be identified with users' investigation and intelligence. This makes it difficult for applications and users to share, retrieve, and analyse the information on the web.

In recent years, the web has evolved to one where both documents and data are linked [1]. The goal of the LOD ${ }^{1}$ community project is publishing and interlinking datasets following the rules [2] for linking data. Hence, a large number of datasets of various domains have been published as Linked Data. Semantic Web was a movement to resolve the difficulties of information retrieval and processing. Moreover, intelligent systems were employed and various intelligent Semantic Web search engines were produced [3]. Although lots of effort was made, many of the problems remained intact. So, Linked Data was introduced for facilitating and removing the existing problems.

\footnotetext{
${ }^{1}$ Linking Open Data

DOI : 10.5121/ijwest.2012.3105
} 
International Journal of Web \& Semantic Technology (IJWesT) Vol.3, No.1, January 2012

Based on the $4^{\text {th }}$ rule of LOD, URIs of datasets' resources are linked to other URIs to discover related things. Various ontologies and schemas such as RDF, RDFS, OWL, SKOS, FOAF, Dublin Core, etc., have defined many typed links that organizations and individuals can use them for publishing their datasets. These links identify the relationship between web resources; for example, rdf:type, dbpedia:country, rdfs:seeAlso, foaf:member, skos:subject, owl:sameAs, dc:type, etc. Some of the links between datasets have been created manually including several links from DBpedia to DBLP, Eurosat, and FactBook. Till now, various approaches and tools [410] have been created which accomplish the interlinking process automatically or semiautomatically. Most of the existing tools and approaches, have concentrated on identifying the same entities in different datasets and moreover some of them require the properties, which should be compared, to be identified for creating the owl:sameAs or others typed links [10].

One of the links employed in Semantic Web is rdfs:seeAlso ${ }^{1}$. This link plays an important role regarding the $4^{\text {th }}$ rule of Linked Data. It has been defined in RDF schema ${ }^{2}$ which relates two resources: subject resource and object resource. $\mathrm{W} 3 \mathrm{C}^{3}$, a major organization that supports the Semantic Web technologies, states that "rdfs:seeAlso is an instance of $r d f$ :property that is used to indicate a resource that might provide additional information about the subject resource" [11]. This definition does not identify the exact relationship and semantic meaning between entities and does not exert any constraint on relationship between resources. As a consequence, the publisher decides which resources should be linked together by rdfs:seeAlso. This link has more history in web and has been widely used in traditional web as well. There are millions of hyperlinks that denoted as seealso which connect web pages; for example, each page of Wikipedia usually contains several seealso links to other pages. While this link performs a great role in linking web pages, some questions arise regarding the existing definition and usages:

1) Where is it come from? (Where is the origin of the link?)

2) If someone decides to interlink a dataset to others manually, how can he identify the resources which provide additional information about one resource? Are there any marks or guidelines that assist him/her?

3) Due to the existing large datasets that contain thousands of resources, manual interlining is time consuming. Therefore, interlinking datasets automatically plays an important role in publishing process. So, referring to the provided general definition, is it possible to design an automated approach that links resources by rdfs:seeAlso? Is it possible to define some patterns which tools may apply them for this purpose?

Answering to the mentioned questions can highly assist data publishers to find suitable target datasets and resources for interlinking, and help researches to design more sophisticated interlinking tools. We will provide answers to these questions by exploring sciences such as information retrieval, library science, etc., and probing their viewpoints in using seeAlso reference following to their correlations and similarities with the Web of Data.

This paper is structured as follows: Section 2 provides a summarized related work. Section 3 gives a comparison between the web technology in one side and library science, and thesauri at the other side. Section 4 describes the roles of seeAlso in linking elements and terms contained in several databases. In section 5, the comprehensiveness of four patterns is analysed and in sections 6 , a summarized conclusion and future work is provided.

\footnotetext{
${ }^{1}$ The abbreviation of seeAlso is $S A$ in our paper and in many corpus

2 www.w3.org/TR/rdf-schema/

${ }^{3}$ World Wide Web Consortium
} 
International Journal of Web \& Semantic Technology (IJWesT) Vol.3, No.1, January 2012

\section{RELATED WORK}

In the Web of Data, each link has a definition that is provided by ontologies and usually has a dereferenceable HTTP address that can be referred to. However, the most attended and discussed relation in Web of Data is owl:sameAs. As defined in W3C, this relation "indicates that two URI references actually refer to the same thing: individuals have the same identity". In various situations in Web of Data, the rdfs:seeAlso link has been used for representing the owl:sameAs relation. It is especially used in linking Friend of a Friend (FOAF) data: it links a FOAF document to another FOAF document that provides additional information.

Regarding to the definition of owl:sameAs provided by $\mathrm{W} 3 \mathrm{C}$, it is possible to interpret several equivalence relations. In [12] the term identity, and the $I S-A$ relation in semantic networks have been argued and discussed and in [13] the different meanings and intents of the IS-A relation have been described. In the Web of Data space, four variations of identity in Linked Data have been introduced [12]: (1) Same thing as but referentially opaque (2) Same thing as but different context (3) Represents, and (4) Very similar to. While the owl:sameAs relation can represent any of these variations in Linked Data, it may be possible to consider other usages and patterns for that. To the best of our knowledge, while there are resources discussed about the owl:sameAs relation, there is no research that opens and describes the rdfs:seeAlso relation in Web of Data.

\section{WORLD WIDE WEB vS. LIBRARY/THESAURUS}

\subsection{Traditional Web vs. Library/Thesaurus}

Traditionally, a library is a large collection of books and nowadays it refers to any collection of resources while the World Wide Web is a very large distributed digital information and data area. The World Wide Web can be compared with a library or thesaurus. They have the following differences:

- Size: The web has more information than a library.

- Structure: while a library has a systematic way for cataloguing information and has a special structure that enables users to find the desired information, the web suffers from its loosely structure nature.

- Referencing/cross-referencing: Libraries contain terms called "controlled vocabularies" which provide a way to organize knowledge of libraries. Some certain uncontrolled terms are used for the sake of pointing to the controlled terms. In web, the pages somewhat can be compared with controlled vocabularies in libraries. However, in traditional web, there is only one type of pointing style; that is, hyperlink which references a page to other page and has not any meaning explicitly. So, it is not possible to differentiate them.

- Searching: unlike most search engines that provide some extra, unnecessary, and useless data and information in response to a user query, libraries deliver more accurate information. Moreover, each of them has its specific search ability and functionality.

- Scope: Some libraries and databases only cover a few domains while the web is a very large distributed database which covers wide range of domains that is published in it.

There are other additional distinctions; i.e., updating, outdated information, interfaces, etc., that are irrelevant to our discussion. 
International Journal of Web \& Semantic Technology (IJWesT) Vol.3, No.1, January 2012

\subsection{Web of Data vs. Library/Thesaurus}

It is evident that two of the main shortcomings of the traditional web compared with the libraries are (1) loss of data structure in publishing process, and (2) non-existence of typed links for connecting web resources. Linked data has suggested rules [2] for solving these problems where arbitrary terms that represent relations are defined (the definitions are available in ontologies) and used for linking web resources. By proper linking, finding the desired information could be accomplished more effective and efficient than before. Using this evolving technology, many libraries have been published on Web of Data, such as Library of Congress Subject Headings, German national Library, British National Bibliography, etc., and the Library Linked Data Group has been set up to gather information on library linked datasets. In many cases, library and Linked Data technology have similar characteristics and design. While the web environment is more open and extensive, the libraries are more abstract and restricted. It seems that the library technology can be assumed as a lower layer of Linked Data (not from architectural view, but from their purpose, applicability, and functionality perspective) and we have the following reasons for that:

- Library science is an earlier technology than the web. Generally, libraries are engaged in collecting books, maps, documents, etc., and in one sense, the web may be assumed as a more extensive and virtual version of libraries.

- Linked Data has many characteristics of libraries that did not exist in traditional web including tightly data structure, and typed referencing to other elements or resources, which are the foundations of Linked Data. There is a shared understanding between the libraries and Linked Data. However, Semantic Web has a different way for conceptualizing data compared with previous library data formats.

- Linked Data provides more appropriate services and capabilities for libraries to be published on the web due to their similar structure. Many institutions have recognized that they should utilize the new technology and publish their data as Linked Data, so making data more reusable and accessible. Therefore, Linked Data acts as a higher level to libraries and provides mechanisms and capabilities that libraries can put to use. Since today, several libraries have been published in web using the Linked Data principles.

- Relationships used in Linked Data are more extensive than the libraries' ones. However, there are many terms that are common.

- In Web of Data, each URI represents and describe an entity, a concept, an individual, or a thing. Mostly, it is the same in libraries where each word or file represents a concept, individual or other elements.

Therefore, we can elicit several similarities and conformities between Linked Data and libraries structure. We believe that it is possible to make use of libraries specifications and definitions to understand and solve some difficulties in Web of Data. We have used this idea for specializing the rdfs:seeAlso link in Web of data due to the fact that this link is also used in several libraries and other comparable structural databases. While rdfs:seeAlso in Web of Data is just defined as providing additional information without any guidelines, libraries and thesauri provide more specific and accurate definitions and patterns for referencing or linking elements that we believe they can be a basis for some usages of rdfs:seeAlso in Web of Data. In the next section, we will identify the role of seeAlso reference in different databases and in different points of view. 


\section{Roles OF SEEALSO}

\subsection{Role of SeeAlso in Pointing to Controlled Vocabularies in Thesauri:}

According to ANSI/NISO [14], Vocabulary control and thesaurus are defined as following: Vocabulary control is used to improve the effectiveness of information storage and retrieval systems, Web navigation systems, and other environments that seek to both identify and locate desired content via some sort of description using language. The primary purpose of vocabulary control is to achieve consistency in the description of content objects and to facilitate retrieval (p. 1)

A thesaurus is a controlled vocabulary arranged in a known order and structured so that the various relationships among terms are displayed clearly and identified by standardized relationship indicators. Relationship indicators should be employed reciprocally. (p. 18)

Controlled vocabularies are used in various knowledge organization systems such as subject headings, thesauri, subject indexing, etc. and applied to different domains such as hospital information systems [15], government information [16], bioinformatics [17], microbiology [18], etc.

According to the [14], there are three types of relationship used between controlled vocabularies:

1) Equivalency

2) Hierarchy

3) Association

Links seeAlso and Related Term ${ }^{l}$ are used between associated terms in controlled vocabulary. Two terms that are associated with each other can be of the following types:

1. Relationships between terms belonging to the same hierarchy:

- Overlapping sibling terms: terms loosely used interchangeably; for example, Ships SA boats.

- Relationships between mutually exclusive sibling terms and the meaning of terms does not overlap; for example, Roses SA daffodils

- Derivational relationships: one of the terms is derived from the other; for example, Parents SA children.

2. Relationships between terms belonging to different hierarchies: Typical relationships shown in table 1 require SA or RT link; for example, Hunting SA hunters.

The mentioned relationships are bidirectional: subject and object terms can be interchanged.

\subsection{Role of SeeAlso in Indexing}

Subject indexing is creating index terms that describe a book or a document and make it easier to find the desired content. Indexer identifies the appropriate terms which are arranged in a systematic order. Creating relationships such as generic relations (IS-A), part-of relations,

\footnotetext{
${ }^{1}$ In this paper and some corpus, the abbreviation of related term is RT
} 
International Journal of Web \& Semantic Technology (IJWesT) Vol.3, No.1, January 2012

subordinate, and superordinate relations between terms, a hierarchical structure is formed and then terms that have a close relation with each other are linked with "related term" reference and terms with distant relation are linked with seeAlso reference [19]. The goal of seeAlso crossreference is establishing a relation between terms which imply to related subjects and provide additional information for user [20]. This definition is also used in other languages, such as Persian language as mentioned in [21].

The seeAlso cross-reference link can be applied to the following conditions:

- Representing a relation between the associative terms [21, 22].

- Representing the antonym relation between terms [21].

- Referencing from genus to species or from category to sub-category [21, 23].

- Representing the whole-part relationship [24] [21] between terms.

- Referencing from a generic term to a specific term [25].

- Referencing from a specific term to a generic term [25].

- Referencing from a term to the preferred term in geographical indexing [21].

Table 1. Association relations between terms belonging to different hierarchies; They are specified in ANSI/NISO standard

\begin{tabular}{|l|l|}
\hline Associative relationship & Example \\
\hline Cause/Effect & Accident/injury \\
\hline Process/Agent & Velocity/measurement/speedometer \\
\hline Process/Counter-agent & Fire/flame retardant \\
\hline Action/Product & Writing/publication \\
\hline Action/Property & Communication/communication skills \\
\hline Action/Target & Teaching/student \\
\hline Concept or Object/Property & Steel alloy/corrosion resistance \\
\hline Concept or Object/Origins & Water/well \\
\hline $\begin{array}{l}\text { Concept or Object / Measurement Unit or } \\
\text { Mechanism }\end{array}$ & Chronometer/minute \\
\hline Raw material/product & Grapes/wine \\
\hline Discipline or Field/Object or practitioner & Neonatology/infant \\
\hline
\end{tabular}

\subsection{Role of SeeAlso in Authority Control}

Authority control is creating and maintaining consistent forms of index terms such as names, titles that used as headings in bibliographic materials. According to [26]:

Authority record is the authorized form of name combined with other information elements that identify and describe the named entity and may also point to other related authority records. (p. 10)

Authority control gives a better understanding of the terms in a catalogue or library. The seeAlso cross referencing is also applied in authority control and is placed between a predecessor name 
International Journal of Web \& Semantic Technology (IJWesT) Vol.3, No.1, January 2012

and an authorized form of a name [26]. This means that a term is referenced to earlier or later form of a term. Library of Congress Subject Headings (LCSH) [27] and Sears List of Subject Heading [28] are authority files for subject headings. Sears list is more suitable for the small public library rather than the Library of Congress Subject Headings. It uses seeAlso reference (1) for making a reference from a general or broader term to a more specific or narrower one; for example, mathematics SA Arithmetic, and (2) for referencing related or associated terms with less or more equal specificity; for example, weather SA climate. It has another uses of SA that are called general references including [28]:

- Common names of different species of a class; for example, flowers SA annuals (also names of flowers such as roses).

- Names of individual persons; for example, artists SA architects (also names of individual artists).

- Names of particular institutions, buildings, societies, etc.; for example, abbeys SA cathedrals (also name of individual abbeys).

- Names of particular geographic features; for example, mountains SA mountaineering (also names of mountain ranges; e.g., Rocky Mountains, etc.)

- Names of places subdivided by subject; for example, population SA birth control (also names of countries, cities, and etc.).

The LCSH was designed originally for representing subjects and describing books, employing controlled vocabulary, provided subject access to bibliographic records that are contained in the Library of Congress Catalogs. It is a large collection of information source that uses certain terms for referencing its subjects including seeAlso and seeAlso from. The first one is used (1) for referencing related or subordinate topic, and (2) for a general reference to an entire group of headings or subdivisions while the second one indicates a related or broader heading which a seeAlso reference is made; for example, epigrams SA quotations (epigrams is the broader term).

In the next section, we provide a summary of the most common usages of seeAlso in information retrieval point of view.

\subsection{The Most Common Usages}

There are different databases in several domains that are available physically or digitally. Each of them may apply various terms for connecting things they contain. Although each has its specific norms for referencing its sources, they usually employ the term seeAlso to link related entities, and concepts and have a common definition for that; i.e., "providing additional information". While databases may interpret it distinct from each other, there are patterns which are more common and are widely used in databases and can be used and attended more in Web of Data. They are as follows:

- Linking a term to another term that is synonym in some way; for example, one city may have another name; i.e., Hegmatane SA Hamedan.

- Linking a term to its narrower term or from a generic term to a specific term; for example, vessel SA boat

- Linking a specific term to its generic or broader term. This pattern is used less compared with the previous one. 
International Journal of Web \& Semantic Technology (IJWesT) Vol.3, No.1, January 2012

- Linking a terms to a subgroup, species, category, phylum, etc.; for example, plant SA Lycophyta.

- Linking a term to its parts representing the whole-part relationship; for example, car SA automobile engine.

- Linking a term to its associative term, or a term to its sibling term; i.e., terms that are in the same level of hierarchy or can be used interchangeably; for example, boat SA ship, and Rose SA daffodil.

While we can specify more semantic relations, the mentioned ones were used more than others in our investigated databases. Hence, they can be more attended in interlinking datasets in Web of Data.

\section{EMPIRICAL STUDY}

In order to determine the universality and comprehensiveness of specified usages, we have done an empirical study of how many times the four patterns, which are shown in table 2, are used in Wikipedia dataset. We have used Wikipedia due to its popularity and extensiveness: it contains entities belonging to various domains and is used as a source of information for several researches. For our purpose, we extracted 120 pages from Wikipedia including various domains such as persons, organizations, concepts, locations, objects, etc., and the pages that are linked to by seeAlso were fetched. The title of pages and four candidate relations were provided to the five expert users. They were asked to identify the implicit semantic relationships between the titles of connected pages, and were asked to match those semantic relationships to the four provided patterns if it is possible. For each of 120 pages, on average 5.62 seeAlso links and totally 675 links have been investigated.

Table 2. Patterns considered in empirical study

\begin{tabular}{|c|}
\hline Relationships \\
\hline Generic to specific relationship \\
\hline Specific to generic relationship \\
\hline Sibling relationship \\
\hline Synonym relationship \\
\hline
\end{tabular}

The results in table 3 show that generic to specific relationship has possessed a significant portion of seeAlso meaning in Wikipedia. It means that providing more detailed and narrower information plays an important role in seeAlso usage. In addition, the four studied patterns constitute about 37 per cent of all seeAlso semantic intentions. Hence, these four patterns can be considered as candidates for semantic meaning of seeAlso in order to linking web resources. 
Table 3. Frequency and density of each pattern

\begin{tabular}{|c|c|c|}
\hline Relationships & $\begin{array}{c}\text { Occurrence } \\
\text { Frequency }\end{array}$ & Density \\
\hline Generic to specific & 175 & $25.92 \%$ \\
\hline Specific to generic & 20 & $2.96 \%$ \\
\hline Sibling relationship & 30 & $4.44 \%$ \\
\hline Synonym relationship & 25 & $3.70 \%$ \\
\hline None & 425 & $62.96 \%$ \\
\hline
\end{tabular}

\section{CONClusions ANd Future Works}

In this paper, we have specified several definitions, usages, and patterns of seeAlso crossreference in libraries, thesauri, subject indexing, and subject headings that can give a great view to the dataset publishers in Linked Data to select the target datasets and resources that provide additional information about their resources and entities. The definitions explained in this paper, constitutes a collection of patterns that each database obeys some elements of that. Moreover, there are undeniable common patterns in databases for referencing purposes demonstrating that we can apply them in Web of Data. In addition, our preliminary empirical study shows promising results for four inspected patterns. It should be mentioned that we do not limit the seeAlso usages in Linked Data by the mentioned use cases. Publishers may employ other models and patterns for recognizing the related resource or even determine several users for distinguishing the related/unrelated ones. However, presenting some well-known and desirable usages that have been widely used will lead organizations and individuals to more efficient and effective dataset publishing.

In the future, we will probe the traditional web more extensively and will investigate more scientific and popular datasets. Moreover, we will not limit our empirical study to only four patterns and will consider more of them in matching step. Additionally, we will conduct and analyse the empirical evidences in the Web of Data to identify the extent of evidences supporting the specified patterns. Moreover, the patterns will be ranked based on their usage's density.

\section{ACKNOWLEDGEMENTS}

This research is supported by the Research Institute for ICT and University of Isfahan. We appreciate anonymous persons who helped by performing the empirical study.

\section{REFERENCES}

[1] C. Bizer, et al., "Linked Data - The Story So Far," International Journal on semantic Web and Information Systems(IJSWIS), vol. 5, pp. 1-22, 2009.

[2] T. B. Lee. (2006, November. 15, 2011). Linked Data [Online]. Available: http://www.w3.org/DesignIssues/LinkedData.html

[3] G. Madhu, et al., "Intelligent Semantic Web Search Engines: A Brief Survey," International journal of Web \& Semantic Technology (IJWesT), vol. 2, 2011. 
International Journal of Web \& Semantic Technology (IJWesT) Vol.3, No.1, January 2012

[4] O. Hassanzadeh and C. Mariano, "Linked Movie Database," in Proc. World Wide Web workshop on Linked Data on the Web, 2009, pp. 194-196.

[5] M. Hausenblas, et al., "Scripting User Contributed Interlinking," in 4th Workshop on Scripting for the Semantic Web (SFSW 08), collocated with the European Semantic Web Conference (ESWC), 2008.

[6] M. Hausenblas, et al., "Interlinking Multimedia: How to Apply Linked Data Principles to Multimedia Fragments," in Linked Data on the Web Workshop (LDOW 09), in conjunction with 18th Int. World Wide Web Conference, Madrid, Spain, 2009.

[7] A.-C. N. Ngomo and S. Auer, "LIMES - A Time-Efficient Approach for Large-Scale Link Discovery on the Web of Data," in International Joint Conferences on Artificial Intelligence (IJCAI), 2011.

[8] Y. Raimond, et al., "Automatic Interlinking of Music Datasets on the Semantic Web," in Proc. Linked Data on the Web Workshop at 17th Int. World Wide Web Conf., Beijing, China, 2008.

[9] F. Scharffe, et al., "RDF-AI: an Architecture for RDF Datasets Matching, Fusion and Interlink," in Proc. IJCAI 2009 workshop on Identity, reference, and knowledge representation (IR-KR), Pasadena, US, 2009.

[10] J. Volz, et al., "Silk - A Link Discovery Framework for the Web of Data," in Proc. 2nd Linked Data on the Web, Madrid, Spain, 2009.

[11] W3C. (2004, November, 15, 2011). RDF Vocabulary Description Language 1.0: RDF Schema [Online]. Available: http://www.w3.org/TR/rdf-schema/

[12] H. Halpin and P. J. Hayes, "When owl:sameAs isn't the Same: An Analysis of IdentityLinks on the Semantic Web," in Proceedings of the WWW2010 workshop on Linked Data on the Web (LDOW), 2010 .

[13] Ronald J. Brachman, "What IS-A Is and Isn't: An Analysis of Taxonomic Links in Semantic Networks," IEEE Computer, vol. 16, pp. 30-36, Oct 1983.

[14] ANSI/NISO, Guidelines for the Construction, Format and Management of Monolingual Controlled Vocabularies, 2005.

[15] A. R. Mori, "Coding systems and controlled vocabularies for hospital information systems," International Journal of Bio-Medical Computing, vol. 39, pp. 93-98, 1995.

[16] A. Craig and S. Schriar, "The Find-It! Illinois controlled vocabulary: Improving access to government information through the Jessica subject tree," Government Information Quarterly, vol. 18, pp. 195 208, 2001.

[17] B. Smith and A. Kumar, "Controlled vocabularies in bioinformatics: a case study in the gene ontology," Drug Discovery Today: BIOSILICO, vol. 2, pp. 246-252, 2004.

[18] T. Korves and M. E. Colosimo, "Controlled vocabularies for microbial virulence factors," Trends in Microbiology, vol. 17, pp. 279-285, 2009.

[19] R. Fugmann, Subject analysis and indexing: theoretical foundation and practical advice vol. Volume 1 of Textbooks for knowledge organization. Frankfurt: Index Verlag, 1993.

[20] L. S. Bonura, The Art of Indexing vol. Volume 13 of Wiley technical communication library. New York: Wiley, 1994.

[21] A. Noruzi, Book Indexing, Second Edition ed. Tehran: Chapar Publication, 2008. 
International Journal of Web \& Semantic Technology (IJWesT) Vol.3, No.1, January 2012

[22] H. H. Wellisch, Glossary of Terminology in Abstracting, Classification, Indexing, and Thesaurus Construction: Information Today, 2000.

[23] H. Borko and C. L. Bernier, Indexing concepts and methods: Academic Press, 1978.

[24] P. Gerstl and S. Pribbenow, "A conceptual theory of part-whole relations and its applications," Data \& Knowledge Engineering, vol. 20, pp. 305-322, November 1996.

[25] P. F. Booth and M. Piggott, Choice and form of entries, 2 ed.: Society of Indexers, 1995.

[26] I. C. o. A. ISAAR, International Standard Archival Authority Record for Corporate Bodies, Persons and Families ISAAR (CPF) Paris: International Council on Archives, 2004.

[27] L. o. Congress, Library of Congress Subject Headings, Ninth edition ed. vol. 2. Washington, 1980.

[28] M. E. Sears and M. T. Mooney, Sears List of Subject Headings, 14th Edition ed.: H. W. Wilson, 1991.

\section{Authors}

Farzam Matinfar received his B.Sc degree in computer engineering from University of Isfahan, Iran in 2004. He received his Master of Software Engineering from University of Isfahan, Iran in 2008. Currently, he is a $\mathrm{PhD}$ candidate in software engineering in Engineering Faculty of University of Isfahan and his research interest includes Semantic Web, Linked Data, Social Web, and E-commerce.

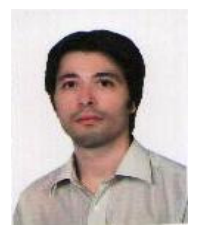

Mohammad Ali Nematbakhsh is an associate professor of computer engineering in the School of Engineering at the University of Isfahan. He received his BSc in electrical engineering from Louisiana Tech University in 1981 and his MSc and $\mathrm{PhD}$ degrees in electrical and computer engineering from the University of Arizona in 1983 and 1987, respectively. He had worked for Micro Advanced Co. and Toshiba Corporation for many years before joining The University of Isfahan. He has published more than 80 research papers, three U.S. registered patents and a database book that is widely used in

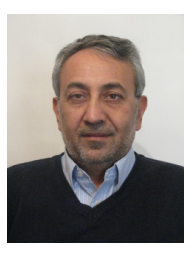
universities. His main research interests include semantics web and computer networks application. 